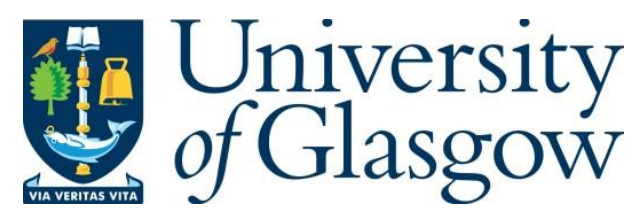

Asuhaimi, F. A., Bu, S. and Imran, M. A. (2019) Joint Resource Allocation and Power Control in Heterogeneous Cellular Networks for Smart Grids. In: IEEE GLOBECOM 2018, Abu Dhabi, United Arab Emirates, 09-13 Dec 2018, ISBN 9781538647271.

There may be differences between this version and the published version. You are advised to consult the publisher's version if you wish to cite from it.

http://eprints.gla.ac.uk/165465/

Deposited on: 21 August 2018

Enlighten - Research publications by members of the University of Glasgow http://eprints.gla.ac.uk 


\title{
Joint Resource Allocation and Power Control in Heterogeneous Cellular Networks for Smart Grids
}

\author{
Fauzun A. Asuhaimi, Shengrong Bu and Muhammad A. Imran \\ Department of Electrical Engineering, University of Glasgow, G12 8QQ, UK. \\ Email: f.abdullah-asuhaimi.1@ research.gla.uk
}

\begin{abstract}
The smart grid communication plays a pivotal role in coordinating energy generation, energy transmission, and energy distribution. Cellular technology with long-term evolution (LTE)-based standards has been a preference for smart grid communication networks. However, conventional cellular networks could suffer from radio access network (RAN) congestion when many smart grid devices attempt access simultaneously. Heterogeneous cellular networks (HetNets) are proposed as important techniques to solve this problem because HetNets can alleviate the RAN congestion by off-loading access attempt from a macrocell to small cells. In smart grid, real-time data from phasor measurement units (PMUs) has a stringent delay requirement in order to ensure the stability of the grid. In this paper, we propose a joint resource allocation and power control scheme to improve the end-to-end delay in HetNets by taking into account the simultaneous transmission of PMUs. We formulate the optimization problem as a mixed integer problem and adopt a game-theoretic approach and the best response dynamics algorithm to solve the problem. Simulation results show that the proposed scheme can significantly minimize the end-to-end delay compared to first-in first-out scheduling and round-robin scheduling schemes.
\end{abstract}

Index Terms-End-to-end delay, heterogeneous cellular network, smart grid communication, phasor measurement units.

\section{INTRODUCTION}

The growing energy demand and greenhouse gas emission problem has led to the shift from traditional power grids to smart grids [1]. The smart grid utilizes a two-way communication between the smart grid domains to increase the reliability of the power grid. The smart grid communication plays an important role in coordinating energy generation, energy transmission and energy distribution, therefore, it is an essential part of an efficient grid control [2]. The smart grid communication can be divided into three kinds of network which are Wide Area Networks (WANs), Neighbourhood Area Networks (NANs) and Home Area Networks (HANs). NANs hold communications at the distribution level which includes transmitting meter and status data to the control center. The data are crucial for many applications such as demand-side management, distribution automation and outage management. Consequently, the performance of smart grid NANs will determine the efficiency of the whole grid.

Of the existing wireless technology, cellular technology with Long-Term Evolution (LTE)-based standards is preferred for NANs due to its high availability and flexibility [3]. Integrating the cellular technology and the smart grid communication network is a significant challenge for the LTE due to the large volume of simultaneous transmission delay-sensitive smart grid data. The simultaneous transmissions from smart grid devices in cellular networks may lead to radio access network (RAN) congestions. Heterogeneous cellular networks (HetNets) are proposed as critical techniques to reduce the RAN congestion because HetNets have the ability to alleviate the RAN congestion by off-loading access attempt from a macrocell to small cells [4]. In HetNets, low-power and lowcost small cell base stations (SCBSs) are deployed to increase the data rate of small cell users (SUEs). Exploiting small cells in cellular networks can also improve the delay in the network by a proper communication technique design.

In smart grids, delay is one of the critical parameters that determine the performance of NANs, especially when distributed power plants based on renewable resource are considered at the distribution level. Distributed renewable energy generations (DEGs) are very dependent on local weather conditions which are highly intermittent. To make control and monitoring possible at energy generation domains, PMUs play a critical role by transmitting real-time dynamic data consisting DEGs' status to the control center. The control center will provide control commands to each PMU based on the status received. However, when data exceed the delay requirement, the control center assumes that the state of a DEG remains unchanged and will not provide the desired control command. This issue may lead to power loss and blackout may occur in severe cases.

There are some works on delay in smart grid communication networks [5]-[7] but no work considers the simultaneous data transmission from PMUs. In this work, we exploit HetNets to reduce the RAN congestion by taking into account the simultaneous transmission of PMUs. In smart grid, PMU measurements are gained by first sampling the voltage and current waveforms through the Global Positioning System and then each sample is time stamped for phase and amplitude variations assessment before sent to the local phasor data concentrator (PDC). All PMUs in a microgrid are synchronized, i.e., measurements are transmitted to the PDC at the same time. This situation may cause severe access contention and leads to excessive delay. A joint resource allocation and power control method can be exploited to improve delay in HetNets for this scenario.

In this paper, we study HetNets for simultaneous transmission of delay-sensitive data from PMUs. The contribution of this paper can be summarized as follows. 


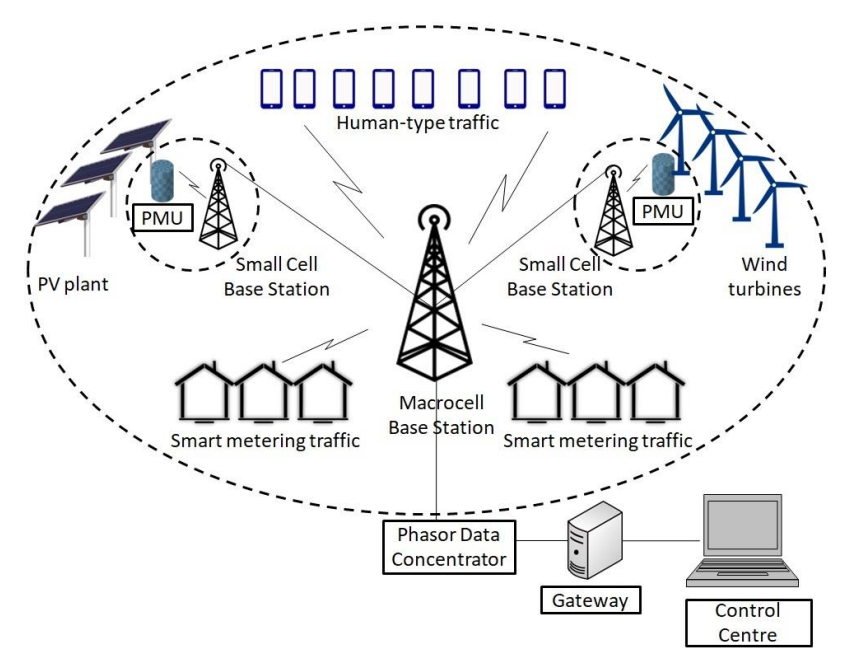

Fig. 1. The communication architecture for the smart grid.

- We exploit HetNets to reduce RAN congestions in smart grid NANs by considering the simultaneous transmission of PMUs.

- We formulate the joint resource allocation and power control problem as a mixed integer problem with design goals to protect the quality-of-service (QoS) of macrocell users (MUEs) and to minimize end-to-end delay of SUEs.

- We adopt a game-theoretic approach and the best response dynamics algorithm to obtain the optimal solution for each SUE in the smart grid environment.

The rest of this paper is organized as follows. The system model is described in Section II. The joint resource allocation and power control problem is formulated in Section III. The optimal power control is derived in Section IV. The gametheoretic analysis and the best response dynamics algorithm for resource optimization are explained in Section V. Simulation results are presented and discussed in Section VI, and the paper is concluded in Section VII.

\section{SySTEM MODEL}

In the HetNet shown in Fig. 1, an orthogonal frequency division multiple access (OFDMA) system is considered. There are one macrocell base station (MBS) and $S$ SCBSs connected to the MBS through wired networks in a service region. Considering the uplink transmissions, PMUs collect the status of DEGs, then transmit the data to the PDC through SCBSs and the MBS. After that, the PDC forwards the data to the control centre in the internet architecture through the gateway in the core network using the wired network as shown in Fig. 1.

\section{A. Heterogeneous Cellular Network Model}

The MBS is located at the center of a cell and it provides a complete coverage over the entire network, while SCBSs are hot spots which offer traffic off-loading, as well as service rate improvement within the macro-edge area. It is assumed that PMUs are located at the edge areas and always connected to SCBSs for a higher service rate. The system is operated in a timeslot manner, where in each timeslot, the spectrum resource licensed to the MBS is divided into multiple subchannels. It is assumed that the MBS is aware of spectrum accessed by SCBSs, and SCBSs can monitor the surrounding radio channel environment and allowed to intelligently access the subchannels.

The MBS serves $M$ MUEs by allocating a subchannel with the same bandwidth to each MUE in each timeslot. At the same time, these $M$ subchannels are shared with $K$ SUEs, as is the common case in real networks. Let the set of MUEs denoted by $\mathcal{M}=\{1, \cdots, M\}$, and $\mathcal{S}=\{1, \cdots, S\}$ denotes a set of SCBSs that are underlaid on the macrocell with each small cell $s \in \mathcal{S}$. The set of SUEs and the set of subchannels are $\mathcal{K}=\{1, \cdots, K\}$ and $\mathcal{N}=\{1, \cdots, N\}$ respectively. Note that a PMU is also a SUE, therefore the SUE refers to the PMU afterwards.

Let $y_{m k, t}^{n} \in\{0,1\}$ denote the subchannel allocation profile of MUE $m$ shared with SUE $k$ at timeslot $t$. Based on the profile, the signal-to-interference-plus-noise ratio (SINR) at SUE $k$ over subchannel $n$ shared with MUE $m$ is given by

$$
\gamma_{k, t}^{n}=\frac{\left|h_{k s}^{n}\right| p_{k, t}}{y_{m k, t}^{n}\left|h_{m s}^{n}\right| p_{m, t}+N_{0}},
$$

where $\left|h_{k s}^{n}\right|$ represents the channel gain between SUE $k$ and SCBS $s$ over subchannel $n$ which can be calculated as $\left|h_{k s}^{n}\right|=C^{s} \zeta_{k s}\left(L_{k s}\right)^{-\alpha} . C^{s}, \zeta_{k s}, L_{k s}$ and $\alpha$ denote the path loss constant, the fast fading component with Nakagami-m distribution, the distance between SUE $k$ and $\operatorname{SCBS} s$, and the path loss exponent respectively. The transmission power of MUE $m$ over subchannel $n$ is denoted by $p_{m}^{n}$ and the variance of the complex Gaussian thermal noise at the receiver represented by $N_{0}$.

The achievable data rate of SUE $k \in \mathcal{K}$ associated to SCBS $s$ over subchannel $n$ at timeslot $t$ is given by [8]

$$
R_{k, t}^{n}=\sum_{\forall n \in \mathcal{N}} \log _{2}\left(1+\frac{\left|h_{k s}^{n}\right|^{2} p_{k, t}}{N_{0}+I_{k, t}^{n}}\right),
$$

where $I_{k, t}^{n}$ indicates the aggregate interference encountered by SUE $k$ which can be calculated as follows [8]

$$
I_{k, t}^{n}=\sum_{\forall m \in \mathcal{M}} y_{m k, t}^{n}\left|h_{m s}^{n}\right|^{2} p_{m, t} .
$$

\section{B. Queue Dynamic of SUE}

All SUEs generate data at the same timeslot. Let $B_{k, t}$ denote the amount of data generated by SUE $k$ at timeslot $t$ which is independent and identically distributed (i.i.d) over timeslots following a uniform distribution $f_{B}(x)$ with an average generation rate $\lambda_{k}$. A first-in-first-out (FIFO) behavior is adopted in the queue model and the generated data is stored in the queue first before transmitted at the next time slot. Let $Q_{k, t}$ denote the queue length of SUE $k$ at timeslot $t$. The queue dynamic of SUE $k$ can be defined as

$$
Q_{k, t+1}=\max \left\{0, Q_{k, t}-R_{k, t}^{n}\right\}+B_{k, t} .
$$


Based on the queue length, the message delay of SUE $k$ can be calculated according to Little's law as follows [9]

$$
D_{k, t}=\frac{Q_{k, t}}{T_{k, t}},
$$

where $T_{k, t}$ denotes the throughput of SUE $k$ at timeslot $t$ which can be expressed as $T_{k, t}=\min \left\{Q_{k, t}, R_{k, t}^{n}\right\}$.

\section{Random Access Procedure in the Preamble Part}

The procedure of random access (RA) consists of two parts which are preamble and message parts. In the preamble part, a user attempts access from the base station (BS) by transmitting a preamble in the RA window [10]. The BS will respond to the request as soon as the preamble is detected by denoting the uplink resource allocation used for the transmission later in the message part. The user will construct a radio resource control connection setup based on the assigned resource and transmits the message in the message part. However, the user will not received any respond from the BS if the preamble is not detected thus it needs to transmit a new preamble in the next RA window.

When two or more users attempt access to the same BS using the same preamble at the same timeslot, a collision occurs and the BS will not detect the request [10]. It is assumed that users will retransmit preamble with equivalent retransmission probability. Let $Z_{l}$ denote the random number which the retransmission takes place after $l$-th collision, with uniform distribution in the range $[1, z]$. It is assumed that $z$ is fixed to 10 timeslots. The access delay of SUE $k$ can be calculated as follows [11]

$$
D_{k}^{A}=D_{0}+\sum_{l=1}^{R}\left[\left(Z_{l}+1\right) T\right],
$$

where $R, T, D_{0}$ denote the number of retransmission probability to successfully transmit the data, the timeslot duration of the system, and the time from the new packet arrival to the beginning of the next time slot respectively. $R$ can be approximated using a geometric distribution with transmission probability $P_{\text {succ }}$ [11], as follows.

$$
P[R=r]=P_{\text {succ }}\left(1-P_{\text {succ }}\right)^{r} .
$$

\section{JOINT RESOURCE ALLOCATION AND POWER CONTROL PROBLEM FORMULATION}

In HetNets, SUEs share subchannels with MUEs, therefore SCBSs need to intelligently allocate subchannels for SUEs and SUEs need to control the transmission power in order to minimize the end-to-end delay. The design goals of the optimization problem are to protect macrocell communications and to exploit the merit of resource sharing between MUEs and SUEs. In the optimization problem, the end-to-end delay can be defined as the time instant from a packet is generated at the source node to the time instant a packet is delivered at the destination node, which includes access and message delays. Considering the RA procedure in the preamble part and the joint resource allocation and power control method in the message part, the end-to-end delay of SUE $k$ can be expressed as follows

$$
D_{k, t}^{\mathrm{end}}=D_{k}^{A}+D_{k, t}\left(y_{m k, t}^{n}, p_{k, t}^{n}\right) .
$$

To minimize the end-to-end delay in HetNets, subchannel allocation and power control schemes are jointly optimized and the problem can be formulated as follows [12]

$$
P 1: \min _{y_{m k, t}^{n}, p_{k, t}, k \in \mathcal{K}} D_{k, t}^{\mathrm{end}}\left(y_{m k, t}^{n}, p_{k, t}\right)
$$

subject to

$$
\begin{aligned}
& C 1: \sum_{k \in \mathcal{K}} h_{k s}^{n} p_{k, t} y_{m k, t}^{n} \leq I_{m}^{t h}, \forall m \in \mathcal{M}, \\
& C 2: \gamma_{k, t}^{n} \geq \gamma_{k}^{\min }, p_{k, t} \leq p_{k}^{\max }, \forall k \in \mathcal{K}, \\
& \text { C3: } \sum_{m \in \mathcal{M}} y_{m k, t} \leq 1, \sum_{k \in \mathcal{K}} y_{m k, t} \leq 1, \forall m \in \mathcal{M}, \forall k \in \mathcal{K} .
\end{aligned}
$$

Let $y_{m k, t}^{n}$ and $p_{k, t}$ denote the optimal resource allocation and the power control for SUE $k$ at timeslot $t$ respectively. Constraint $C 1$ limits the interference caused by SUE $k$ over subchannel $n$ occupied by MUE $m$ below a threshold $I_{m}^{\text {th }}$ to protect the QoS of MUE $m$. The next constraint indicates a minimum SINR requirement, $\gamma_{k}^{\min }$ and a maximum transmission power for each SUE, $p_{k, t}^{\max }$. The last constraint limits the reuse of subchannel to one user only and each SUE can only reuse only one subchannel in a timeslot.

The formulated problem $P 1$ has a mixed integers nature, therefore it is difficult to address. Therefore, the optimal power control is obtained under a fixed resource allocation profile. Then the optimal resource allocation problem can be constructed using a game-theoretic approach. Lastly, the best response dynamic algorithm is adopted to obtain the optimal solution.

\section{Optimal Power CONTROL}

It is assumed that the subchannel allocation for each SUE has been preset under constraint $C 3$ and fixed for all $k$ and $m$. Problem $P 1$ is then converted into a power control problem, given as follows

$$
P 2: \min _{p_{k, t}, k \in \mathcal{K}} D_{k, t}^{\mathrm{end}}\left(p_{k, t}\right)
$$

subject to

$$
\begin{aligned}
& C 1: \sum_{k \in \mathcal{K}} h_{k s}^{n} p_{k, t} y_{m k, t}^{n} \leq I_{m}^{t h}, \forall m \in \mathcal{M}, \\
& C 2: \gamma_{k, t}^{n} \geq \gamma_{k}^{\min }, p_{k, t} \leq p_{k}^{\max }, \forall k \in \mathcal{K} .
\end{aligned}
$$

To obtain the optimal power control in $P 2$, the characteristic of the feasible region under constraints $C 1$ and $C 2$ is investigated, with the assumption that SUE $k \in \mathcal{K}$ shares the subchannel occupied by MUE $m \in \mathcal{M}$. To achieve both lower and upper bounds of $p_{k, t}$, the transmission power in $C 1$ is set to 0 and equation (1) is substituted in $C 2$, given as follows [12] 


$$
\left\{\begin{aligned}
p_{k, t}^{\min } & =\frac{\gamma_{k, t}^{\min }\left(h_{m s}^{n} p_{m, t}+N_{0}\right)}{h_{k s}^{n}} \\
p_{k, t}^{\mathrm{th}, \max } & =\frac{I_{m}^{t h}}{h_{k 0}^{n}} .
\end{aligned}\right.
$$

Here, the index 0 in $h_{k 0}^{n}$ refers to the MBS. Then the restricted distance between the MUE and the SUE over the same subchannel is investigated. The distance between MUE $m$ and SCBS $s$ associated for SUE $k, L_{m s, t}$ needs to satisfy the following equation [12]

$$
L_{m s, t} \geq L_{m s}^{\min }=\sqrt[\alpha]{\frac{\min \left\{p_{k, t}^{t h, \max }, p_{k}^{\max }\right\} h_{k s}^{n}-\gamma_{k}^{\min } N_{0}}{C^{s} \zeta_{m s} \gamma_{k}^{\min } p_{m, t}}} .
$$

Therefore, the optimal solution to $P 2$ denoted by $p_{k, t}^{\text {opt }}$, and the optimal objective function can be calculated as follows

$$
\left\{\begin{aligned}
p_{k, t}^{\mathrm{opt}} & =\min \left\{\frac{I_{m}^{t h}}{h_{k 0}}, p_{k}^{\max }\right\} \\
D_{k, t}^{\mathrm{end}}\left(p_{k, t}\right) & =D_{k}^{A}+\frac{Q_{k, t}}{\log _{2}\left(1+\frac{h_{k s}^{n} p_{k, t}}{I_{k}^{n} N_{0}}\right)}
\end{aligned}\right.
$$

\section{GAME-THEORETIC ANALYSIS AND BEST RESPONSE DYNAMICS ALGORITHM FOR RESOURCE OPTIMIZATION}

The optimal resource allocation for all $k \in \mathcal{K}$ is investigated based on the obtained optimal power control, $p_{k, t}^{\text {opt }}$ which can be expressed as follows [12]

$$
P 3: \min _{y_{m k}^{n}, k \in \mathcal{K}} D_{k, t}^{\mathrm{end}}\left(y_{m k}^{n}, p_{k, t}^{\mathrm{opt}}\right)
$$

subject to

$$
C 1: \sum_{m \in \mathcal{M}} y_{m k}^{n} \leq 1, \sum_{k \in \mathcal{K}} y_{m k}^{n} \leq 1, \forall m \in \mathcal{M}, \forall k \in \mathcal{K}
$$

A game-theoretic approach is exploited in order to obtain the resource optimization. Therefore, problem $P 3$ is transformed into an unconstrained game problem $P 4$.

\section{A. Game Formulation}

In the game-theoretic approach, strategy and available strategy sets can be defined as the set of all possible resource allocation for the players, and the allowable selection set of a certain player when the location constrained for each player has been determined, respectively.

1) The strategy set: All SUEs in the cell are the players in the game model, $j=k, \forall k \in \mathcal{K}$. The action of $j$ is given by $\mathbf{a}_{j}=\left(y_{1 k}^{n}, y_{2 k}^{n}, \cdots, y_{M k}^{n}\right)^{T}$ where $\mathbf{a}_{j} \in \mathcal{A}_{j}=\left\{\mathbf{e}_{1}, \mathbf{e}_{2}, \cdots, \mathbf{e}_{M}\right\}$. The vector $\mathbf{e}_{n}=\left(e_{n 1}, e_{n 2}, \cdots, e_{n M}\right)^{T} \in \mathbb{M}^{M}$ denotes the $n$ th vector of the canonical base spanning the space of real vector of dimension $M$, i.e., $e_{n n}=1$ and $e_{n n^{\prime}}=0, \forall n^{\prime} \in \frac{\mathcal{M}}{\{n\}}$. The subchannel allocation profile for all players denoted by $\mathbf{a}=\left(\mathbf{a}_{1}, \mathbf{a}_{2}, \cdots, \mathbf{a}_{K}\right)$ and the space of all possible assignment denoted by $\mathcal{A}=\mathcal{A}_{1} x \mathcal{A}_{2} x \cdots x \mathcal{A}_{K}$.
2) The available strategy set: $\mathcal{A}_{j}$ is the general strategy set for player $j, \forall j \in \mathcal{J}$, which does not consider the subchannel allocation constrain and the location-based restriction of players. Some action strategies in $\mathcal{A}_{j}$ are unavailable due to these reasons when the restriction is given. Let $\mathbf{x}\left(\mathbf{a}_{j}\right)=x_{1}\left(\mathbf{a}_{j}\right), x_{2}\left(\mathbf{a}_{j}\right), \cdots, x_{M}\left(\mathbf{a}_{j}\right)$ denote the condition index of subchannels, which contains the information of available subchannels for player $j$ by taking into account the restriction $C 1$ in (17), which acknowledge by $\mathcal{A}_{j}$. Let $x_{n}\left(\mathbf{a}_{j}\right)$ denote the set of SUEs except $j$ occupied the subchannel $n$. The map of restrained strategy set of player $j$ based on $\mathcal{A}_{j}$ and $\mathbf{x}\left(\mathbf{a}_{j}\right)$ is given as $\overline{\mathcal{A}}_{j}=\mathcal{A}_{j}\left\{\mathbf{e}_{n}: x_{n}\left(\mathbf{a}_{j}\right) \bigcap \mathcal{K} \neq \emptyset \bigcup\left|x_{n}\left(\mathbf{a}_{j}\right)\right|=1, \forall n \in\right.$ $\mathcal{M}$ \}. The restrained action space of all players under constrain $C 1$ in (17) denoted by $\overline{\mathcal{A}}=\overline{\mathcal{A}}_{1} \mathrm{x} \overline{\mathcal{A}}_{2} \mathrm{x}, \cdots, \overline{\mathcal{A}}_{K}$.

The available strategy set $\Delta\left(\overline{\mathcal{A}}_{j}\right)$ of player $j$ by means of $\overline{\mathcal{A}}_{j}$ with location restriction can be expressed as follows

$$
\triangle\left(\overline{\mathcal{A}}_{j}\right)=\left\{\mathbf{e}_{n}, \forall n \in \Lambda(\overline{\mathcal{M}}\}\right.
$$

in which $\overline{\mathcal{M}}=\left\{n: \mathbf{e}_{n} \in \overline{\mathcal{A}}_{j}, \forall n \in \mathcal{M}\right\}$ and $\Lambda(\overline{\mathcal{M}})=n$ : $\left.L_{m s_{j}} \geq L_{m s_{j}, \min }, \forall n \in \overline{\mathcal{M}}\right\}$. The utility for unavailable strategy action is assigned to an extremely small value (0) for the decoupling of strategy set, which result in the conversion of problem $P 3$ to an unconstrained optimization problem $P 4$.

$$
P 4: \min _{\mathbf{a} \in \mathcal{A}} D_{j}^{\mathrm{end}}(\mathbf{a})=\sum_{j \in \mathcal{J}} D_{j}^{\mathrm{end}}(\mathbf{a}) y_{\left\{\mathbf{a}_{j} \in \Delta\left(\overline{\mathcal{F}}_{j}\right)\right\}},
$$

where $D_{j}^{\text {end }}(\mathbf{a})=D_{j}^{A}+\frac{Q_{t}}{\min \left\{Q_{t}, R_{j}(\mathbf{a})\right\}}$ and $R_{j}(\mathbf{a})=$ $\sum_{m \in \mathcal{M}} \log _{2}\left(1+\gamma_{k}^{n}\left(p_{k}^{\mathrm{opt}}\right)\right) y_{m k}^{n}, k \in \mathcal{K}$.

Problem $P 4$ can be constructed as a potential game model [12]. The potential game model is extremely useful for effi-

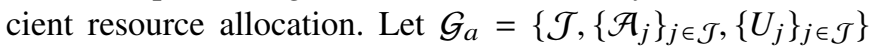
denote the utility function of the game model. Due to the coupling among strategy sets, the expression of utility function cannot be derived, but the difference when player $j$ changes the strategy from $\mathbf{a}_{j}$ to $\mathbf{a}_{j}^{\prime}$ can be obtained as $\hat{U}_{j}\left(\mathbf{a}_{j}^{\prime}, \mathbf{a}\right)=$ $U_{j}\left(\mathbf{a}_{j}^{\prime}, \mathbf{a}\right)-U_{j}\left(\mathbf{a}_{j}, \mathbf{a}\right)=\sum_{l \in \mathcal{J}}\left(u_{l}\left(\mathbf{a}_{j}^{\prime}\right)-u_{l}\left(\mathbf{a}_{j}\right)\right) y_{l \in \overline{\mathcal{J}}\left(\mathbf{a}_{j}^{\prime}, \mathbf{a}\right)}$. Locations of nonzero elements of the action vector represented by the set $\overline{\mathcal{J}}\left(\mathbf{a}_{j}^{\prime}, \mathbf{a}\right)=\left\{j, x_{\zeta\left(\mathbf{a}_{j}\right)}\left(\mathbf{a}_{j}\right), x_{\zeta\left(\mathbf{a}_{j}\right)}\left(\mathbf{a}_{j}^{\prime}\right)\right\}$ and $\zeta($.

\section{B. Best Response Dynamics Algorithm}

The best respond dynamics algorithm [12] is adopted to find a Nash equilibrium (NE) point of $\mathcal{G}_{a}$ which is the global optimal solution for problem $P 1$. In the game model $\mathcal{G}_{a}$, at least one NE exists which minimizes the objective function of $P 3$ [12]. In problem $P 4$, the optimum can be obtained by getting the difference of utilities for each player when the strategy changed. Therefore, to obtain the NE, the best response rule [12] is applied to solve $\mathcal{G}_{a}$ by sharing information between players of selecting the same subchannel, as described in Algorithm 1. This rule can converge to a NE point of the game as well as finds the best NE. Therefore the optimal subchannel allocation profile for HetNets with constraint QoS requirements can be achieved by Algorithm 1. The complexity for one iteration in Algorithm 1 is $O(|J|(M-S+2))$ [12]. 


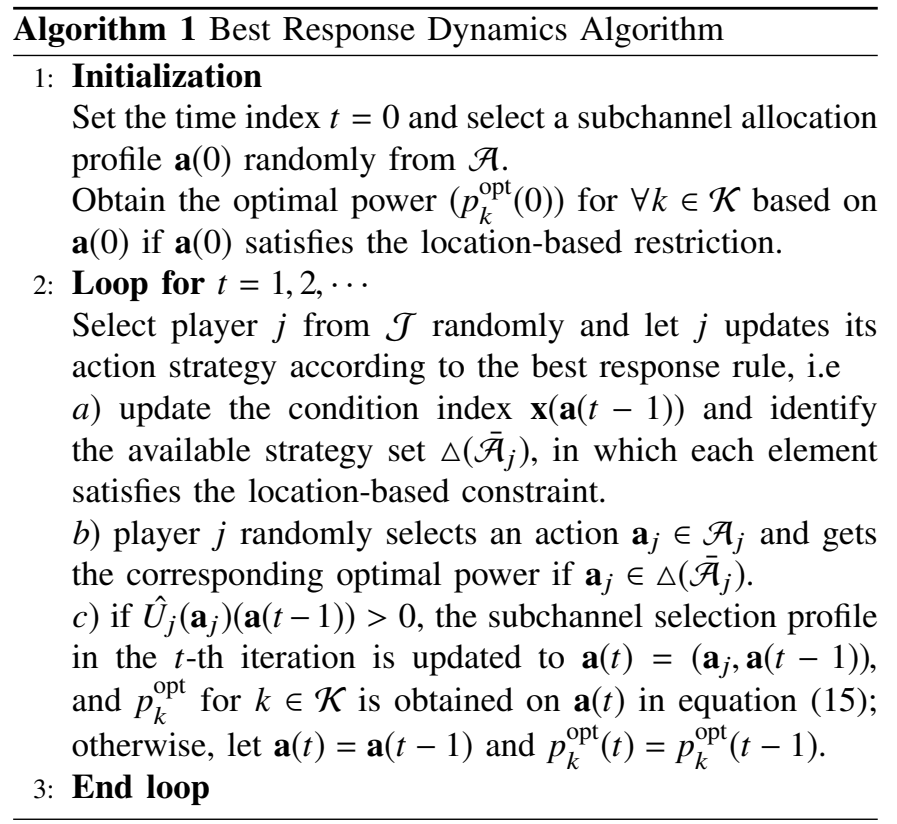

TABLE I

SIMULATION PARAMETERS

\begin{tabular}{lr}
\hline Macrocell radius & $400 \mathrm{~m}[8]$ \\
\hline Small cell radius & $50 \mathrm{~m}[8]$ \\
\hline Number of MUEs & $30[8]$ \\
\hline Number of SUEs & $15[8]$ \\
\hline Number of subchannel & 30 \\
\hline Maximum SINR requirement of SUEs $\left(\gamma_{k}^{\mathrm{min}}\right)$ & $20 \mathrm{~dB}[12]$ \\
\hline Minimum interference threshold of MUEs $\left(I_{m}^{t h}\right)$ & $10^{-6}[12]$ \\
\hline Thermal noise $\left(N_{0}\right)$ & $-174 \mathrm{~dB}[12]$ \\
\hline Path loss constant $\left(C^{s}\right)$ & $10^{-2}[12]$ \\
\hline Path loss exponent $(\alpha)$ & $4.8[12]$ \\
\hline Bandwidth of each subchannel & $180 \mathrm{kHz}[3]$ \\
\hline Maximum transmission power of SUE & $14 \mathrm{dBm} \mathrm{[3]}$ \\
\hline
\end{tabular}

\section{Simulation RESUltS AND DisCUSSION}

Computer simulations are used to evaluate the performance of the proposed scheme. System parameters are explained and simulation results are discussed in this section.

\section{A. System Parameters}

Each PMU has an average generation rate of 1 packet/s with the size of each packet is 102 Bytes for $50 \mathrm{~Hz}$ reporting state [13]. $P_{\text {succ }}$ is 0.85 [11] and the length of each timeslot is $1 \mathrm{~ms}$. In this system, the number of SUEs is fixed while the number of MUEs is varied. A detailed list of simulation parameters are given in Table I. These values are used in the sequel, unless otherwise specified.

Firstly, the performance of the HetNet is compared with the cellular network in terms of the number of preamble collision for various total number of simultaneous access attempts by users using the RA procedure. The cellular network refers to the network with only one serving BS in a macrocell. Fig. 2 shows that at the same total number of attempts, the HetNet gives a significant collision reduction due to its offload capability, consequently proves that HetNets can reduce the RAN congestion. Then, the demonstration of the algorithm

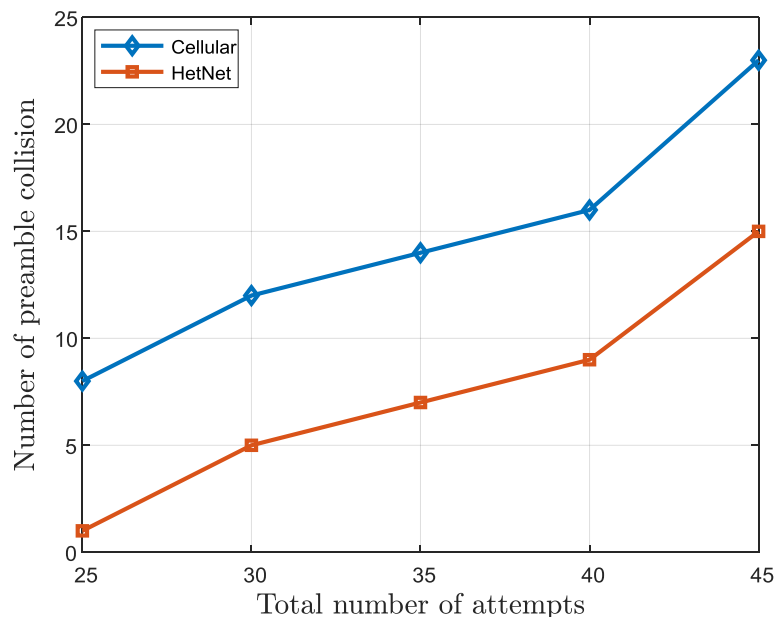

Fig. 2. Number of preamble collision with various total number of attempts.

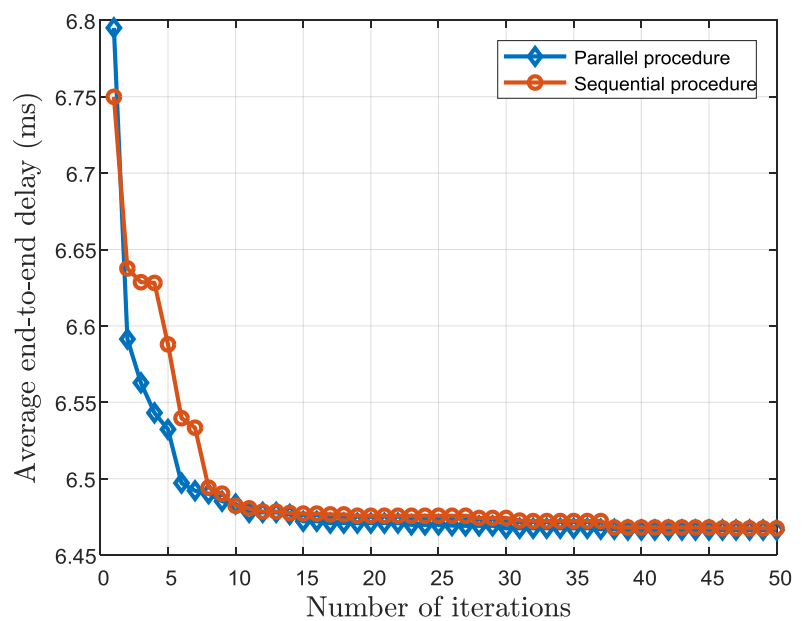

Fig. 3. Convergence of the Nash equilibrium using the Best Response Dynamics Algorithm.

to obtain the NE using parallel and sequential procedures [14] is hold. Fig. 3 shows that the parallel procedure converges faster than the sequential procedure. This is because in the parallel procedure, the SUEs can try to find their best-response decision simultaneously. Moreover, the result shows that the NE can be obtained using the algorithm. Next, the impact of the minimum interference thresholds of MUEs to the average end-to-end delay of SUEs for different maximum transmission power of SUEs is studied. Fig. 4 shows that as the interference increases, the average end-to-end delay increases. The reason for that is because when the interference increases, SUEs will try to decrease the transmission power, which result in a lower data rate. Low data rate leads to high end-to-end delay, as according to Little's law.

Finally, the performance of the proposed scheme is compared with first-in first-out (FIFO) and round-robin (R-R) scheduling schemes [15] in terms of the average end-to-end delay. In the FIFO scheme, the BS allocates subchannels based on a first-come first-served basis, while in the R-R 


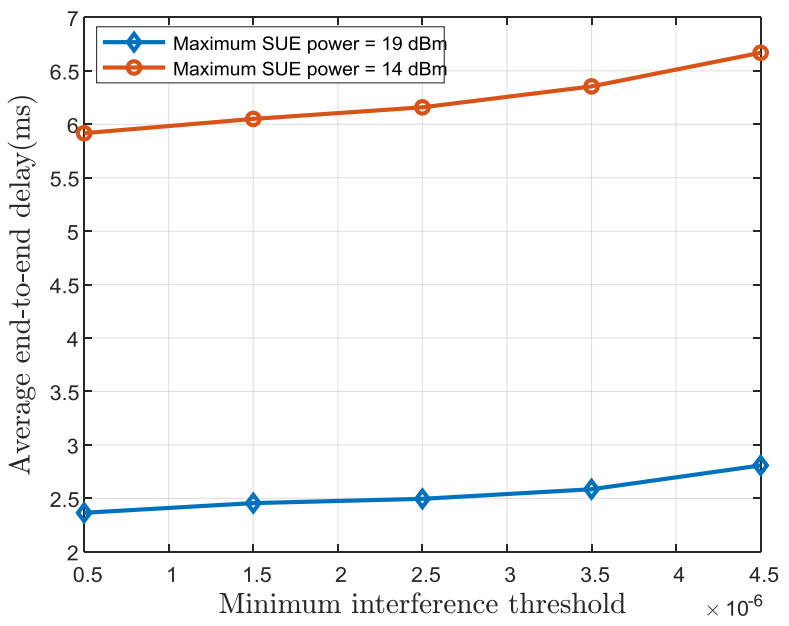

Fig. 4. The average end-to-end delay with various minimum interference threshold.

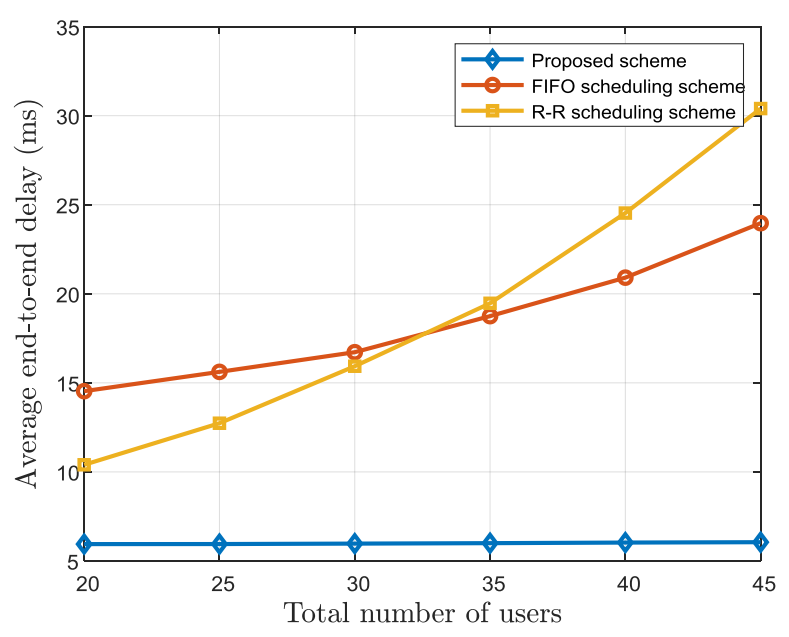

Fig. 5. The average end-to-end delay with various total number of users.

scheduling scheme, the MBS tries to reduce collisions by scheduling users one-by-one before off-loading them to the corresponding SCBSs. As a result, the average end-to-end delay for both schemes are increasing when increasing the number of users in a cell. The reason for that is because in the FIFO scheme, the increase of users will increase the collisions, while in R-R scheme the increase of users will increase the number of timeslot for a complete scheduling cycle. Moreover, the R-R scheduling scheme produces highest delay when the number of users more than 33 , as this scheme is not effective for a large number of users with light traffic [15]. In the proposed scheme, the change in the total number of users does not give a significant impact to the average end-to-end delay, and at the same total number of users, the proposed scheme outperforms other two schemes as shown in Fig. 5.

\section{CONCLUSION}

This paper studied HetNets for simultaneous transmissions of delay-sensitive PMU data. A joint resource allocation and power control method was proposed to minimize the end-to-end delay in HetNets. The optimization problem was formulated as a mixed integer problem, and a game-theoretic approach and the best response algorithm were adopted to solve the problem. Simulation results showed that in the RA procedure, HetNets has lower preamble collisions as the total number of attempts increases compared to cellular networks. Additionally, the proposed scheme gives a significant delay improvement when the total number of users increases compared to cellular and R-R scheduling schemes. In future, the study on the impact of the PMUs' end-to-end delay in HetNets to the total power loss in grids can be held.

\section{REFERENCES}

[1] H. Farhangi, "The path of the smart grid," IEEE power and energy magazine, vol. 8, no. 1, pp. 18-18, Jan. 2010.

[2] T. Sauter and M. Lobashov, "End-to-end communication architecture for smart grids," IEEE Transactions on Industrial Electronics, vol. 58, no. 4, pp. 1218-1228, 2011.

[3] Y. Cao, T. Jiang, M. He, and J. Zhang, "Device-to-device communications for energy management: A smart grid case," IEEE Journal on Selected Areas in Communications, vol. 34, no. 1, pp. 190-201, Jan. 2016.

[4] N. Xia, H.-H. Chen, and C.-S. Yang, "Radio resource management in machine-to-machine communications-a survey," IEEE Communications Surveys \& Tutorials, 2017.

[5] R. Pourramezan, Y. Seyedi, H. Karimi, G. Zhu, and M. Mont-Briant, "Design of an advanced phasor data concentrator for monitoring of distributed energy resources in smart microgrids," IEEE Transactions on Industrial Informatics, vol. 13, no. 6, pp. 3027-3036, 2017.

[6] Y. Seyedi, H. Karimi, and J. M. Guerrero, "Centralized disturbance detection in smart microgrids with noisy and intermittent synchrophasor data," IEEE Transactions on Smart Grid, vol. 8, no. 6, pp. 2775-2783, 2017.

[7] P.-Y. Kong, C.-W. Liu, and J.-A. Jiang, "Cost-efficient placement of communication connections for transmission line monitoring," IEEE Transactions on Industrial Electronics, vol. 64, no. 5, pp. 4058-4067, 2017.

[8] S. Samarakoon, M. Bennis, W. Saad, and M. Latva-aho, "Backhaulaware interference management in the uplink of wireless small cell networks," IEEE Transactions on Wireless Communications, vol. 12 , no. 11, pp. 5813-5825, 2013

[9] L. Lei, Y. Kuang, N. Cheng, X. S. Shen, Z. Zhong, and C. Lin, "Delayoptimal dynamic mode selection and resource allocation in device-todevice communicationspart i: Optimal policy," IEEE Transactions on Vehicular Technology, vol. 65, no. 5, pp. 3474-3490, 2016.

[10] A. Biral, M. Centenaro, A. Zanella, L. Vangelista, and M. Zorzi, "The challenges of $\mathrm{m} 2 \mathrm{~m}$ massive access in wireless cellular networks," Digital Communications and Networks, vol. 1, no. 1, pp. 1-19, 2015.

[11] M. E. Rivero-Angeles, D. Lara-Rodríguez, and F. A. Cruz-Pérez, "Differentiated backoff strategies for prioritized random access delay in multiservice cellular networks," IEEE Transactions on Vehicular Technology, vol. 58, no. 1, pp. 381-397, 2009.

[12] H. Dai, Y. Huang, R. Zhao, J. Wang, and L. Yang, "Resource optimization for device-to-device and small cell uplink communications underlaying cellular networks," IEEE Transactions on Vehicular Technology, 2017.

[13] K. V. Katsaros, B. Yang, W. K. Chai, and G. Pavlou, "Low latency communication infrastructure for synchrophasor applications in distribution networks," in Smart Grid Communications (SmartGridComm), 2014 IEEE International Conference on. IEEE, 2014, pp. 392-397.

[14] F. Wang, M. Krunz, and S. Cui, "Price-based spectrum management in cognitive radio networks," IEEE Journal of selected topics in signal processing, vol. 2, no. 1, pp. 74-87, 2008.

[15] Y. Zhong, T. Q. Quek, and X. Ge, "Heterogeneous cellular networks with spatio-temporal traffic: Delay analysis and scheduling," IEEE Journal on Selected Areas in Communications, vol. 35, no. 6, pp. 1373 1386, 2017. 